PROCEEDINGS OF THE

AMERICAN MATHEMATICAL SOCIETY

Volume 137, Number 7, July 2009, Pages 2437-2440

S 0002-9939(09)09802-5

Article electronically published on January 30, 2009

\title{
CLASSIFICATION OF ALMOST QUARTER-PINCHED MANIFOLDS
}

\author{
PETER PETERSEN AND TERENCE TAO
}

(Communicated by Richard A. Wentworth)

\begin{abstract}
We show that if a simply connected manifold is almost quarterpinched, then it is diffeomorphic to a CROSS or a sphere.
\end{abstract}

\section{INTRODUCTION}

The goal of this paper is to use the new results of Brendle and Schoen in [5], 6] to sharpen some older results about almost quarter-pinched manifolds studied by Berger and Abresch-Meyer in [3, 2]. The main goal is to show that there can't be exotic spheres which are almost quarter-pinched. More precisely, we prove

Theorem 1.1. There exist $\varepsilon(n)>0$ so that any simply connected $n$-dimensional Riemannian manifold $(M, g)$ with sectional curvatures in $[1 / 4-\varepsilon, 1]$ is diffeomorphic to a sphere or a compact rank one symmetric space (CROSS).

In [3], Berger established this result in even dimensions, but with the weaker conclusion that $M$ was either homeomorphic to a sphere or diffeomorphic to a CROSS (see also [9] for other results in this direction). To prove his result, Berger considered a sequence of almost quarter-pinched manifolds; then using Cheeger-Gromov compactness theory, he passed to a limit that was weakly quarter-pinched in the sense of comparison theory. It does not seem that a similar strategy would work for Theorem [1.1 the difficulty is that there is no obvious way to use comparison curvatures to get information about isotropic curvatures on manifolds that only have weakly defined curvature tensors. Instead we apply the Ricci flow to each of the manifolds in the sequence and show that in the limit we get a Ricci flow, where we have nice metrics for positive time. These are not necessarily quarter-pinched (as this property is not preserved by Ricci flow), but they do have nonnegative isotropic curvatures when we add a factor of $\mathbb{R}^{2}$ to the metric. Thus we can use the classification in [6] to understand what the candidates for limit manifolds are. Finally it is worth mentioning that there is at least one example of an exotic sphere with positive sectional curvature (see [14]).

All results in Riemannian geometry which are not specifically referenced can be found in [13] and similarly for results on the Ricci Flow in [7] and [8].

Received by the editors July 11, 2008, and, in revised form, October 16, 2008.

2000 Mathematics Subject Classification. Primary 53C21.

(C)2009 American Mathematical Society 


\section{Proof of Theorem 1.1}

Assume by contradiction that Theorem 1.1 failed in some fixed dimension $n$. Then one could find a sequence of simply connected $n$-dimensional Riemannian manifolds $\left(M_{i}, g_{i}\right)$ such that $1 / 4-1 / i \leq \sec \leq 1$, and such that none of the $M_{i}$ were diffeomorphic to a sphere or a CROSS.

In even dimensions it is a classical result of Klingenberg that such manifolds have injectivity radius $\geq \pi$. In odd dimensions this is far more subtle and was settled by Abresch and Meyer in [1. The fact that there is a uniform lower bound for the sectional curvature shows that the diameter is also bounded, by Myers' theorem. Finally, the sectional curvature bounds imply that the curvature tensors of $g_{i}$ are uniformly bounded. By the standard local existence theory for Ricci flow (10, 11, and [7), we can thus run the Ricci flow for a fixed amount of time $\left[0, t_{0}\right]$ for each of these metrics $\left(M_{i}, g_{i}\right)$, with the curvature tensor uniformly bounded in $i$ on this time interval.

Putting all this together, we obtain a family of Ricci flows $\left(M_{i}, g_{i}(t), t \in\left[0, t_{0}\right]\right)$ with uniformly bounded geometry. Hamilton's extension of the Cheeger-Gromov compactness theorem then guarantees us that some subsequence converges to a Ricci flow $\left(M, g(t), t \in\left[0, t_{0}\right]\right)$ ([12] and [8]); the convergence is only in the $C^{1, \alpha}$, $\alpha<1$ sense at time $t=0$, but is in the $C^{\infty}$ sense for $0<t \leq t_{0}$.

We cannot expect $g(t)$ to be quarter-pinched for $t>0$, and when $t=0$ the metric isn't sufficiently smooth that this makes sense. However, for small $t$ the metrics $g_{i}(t)$ have sectional curvature $\geq 1 / 4-1 / i-C(n) t$ for an absolute constant $C>0$ (see [15, Proposition 2.5]). As the metrics $g_{i}(t)$ converge in the $C^{\infty}$ topology for $t>0$ we see that $g(t)$ has positive sectional curvature for $t>0$. This shows that the metric is irreducible. Below we will show that the product metric on $M \times \mathbb{R}^{2}$ has nonnegative isotropic curvature for $t>0$. The Brendle-Schoen classification (see [6. Theorem 2]) of such metrics then shows that $M$ and hence $M_{i}$ (for sufficiently large $i$ ) are diffeomorphic to a sphere or compact rank one symmetric space, giving the required contradiction.

In Brendle-Schoen 5 the authors show that a quarter-pinched metric also has isotropic curvature even after the metric has been multiplied with the factor $\mathbb{R}^{2}$. The proof is entirely algebraic and also shows that if the metric is almost quarterpinched, then there is a small lower bound for the isotropic curvatures, again even after we have added the $\mathbb{R}^{2}$ factor. Thus the metrics $\left(M_{i}, g_{i}(0)\right)$ have a lower bound $-\varepsilon_{i}$ for the isotropic curvatures on $M_{i} \times \mathbb{R}^{2}$, where $\varepsilon_{i} \rightarrow 0$ as $i \rightarrow \infty$. (The $\varepsilon_{i}$ can in fact be taken to be a multiple of $\frac{1}{i}$, but this is not important for the proof.)

Finally we need to estimate the lower bound for the isotropic curvatures of $g_{i}(t)$ on $M_{i} \times \mathbb{R}^{2}$. It is a general fact that they will be bounded from below by $-\varepsilon_{i} \exp (C t)$, where $C$ is a constant that depends only on the curvature bounds for $g_{i}(0)$. This was established by Hamilton in the proof of [11, Theorem 4.3] as a general property of solutions to heat flows. Specifically consider a heat flow

$$
\partial_{t} T=\Delta T+\phi(T)
$$

on tensors $T$ and a continuous, compact, and convex condition $X$ on the tensors $T$ that is also invariant under the natural $O(n)$ action induced on tensors and future invariants under the ODE $\partial_{t} T=\phi(T)$. Hamilton shows that $X$ is then also invariant under the heat flow by showing that if a solution to the heat flow starts out at some distant $\varepsilon>0$ from $X$, then at time $t$ it will be at most distance 
$\varepsilon \exp (C t)$ away from $X$. Here $C$ depends on the set $X$ and $\phi$. The condition $X$ is rarely compact in applications, but this can easily be finessed by modifying the PDE and ODE, as we shall now discuss. In our situation we are considering the evolution of the curvature tensor

$$
\partial_{t} R=\Delta R+R^{2}+R^{\#}
$$

under the Ricci flow. Initially the curvature tensor is bounded by some fixed constant $K_{1}$ that depends only on pinching constants and dimension. This shows that the flow exists on some fixed time interval $\left[0, t_{0}\right]$ and that the curvature tensors are bounded by some other controlled constant $K_{2}$ on this interval. If we have a continuous, convex, $O(n)$, and future ODE invariant condition $X$, then we can intersect it with the compact, convex, and $O(n)$ invariant set: $|R| \leq K$, where $K>K_{2}$. Next we modify the vector field $\phi(R)=R^{2}+R^{\#}$ outside the region $|R| \leq K_{2}$ to make the new condition invariant under the ODE $\partial_{t} R=R^{2}+R^{\#}$. As long as we only consider initial conditions where the curvature is bounded by $K_{1}$ the solutions will not be affected by these changes.

Finally we can now use that for $t>0$ the metrics $g_{i}(t)$ converge to $g(t)$ in the $C^{\infty}$ topology to see that the limit metrics $g(t)$ have nonnegative isotropic curvatures on $M \times \mathbb{R}^{2}$, as desired. The proof of Theorem 1.1 is now complete.

\section{ACKNOWLEDGMEnTs}

The first author would like to thank Frederick Wilhelm for bringing the above question to our attention. The second author is supported by NSF grant DMS0649473 and a grant from the MacArthur Foundation.

\section{REFERENCES}

[1] U. Abresch and W.T. Meyer, Pinching below 1/4, injectivity radius, and conjugate radius. J. Differential Geom. 40 (1994), no. 3, 643-691. MR1305984 (95j:53053)

[2] U. Abresch and W.T. Meyer, A sphere theorem with a pinching constant below 1/4. J. Differential Geom. 44 (1996), no. 2, 214-261. MR1425576 (97i:53036)

[3] M. Berger, Sur les variétés riemanniennes pincées juste au-dessous de 1/4. Ann. Inst. Fourier (Grenoble) 33 (1983), no. 2, 135-150. MR699491 (85d:53017)

[4] C. Böhm and B. Wilking, Manifolds with positive curvature operators are space forms. Ann. of Math. (2) 167 (2008), 1079-1097.

[5] S. Brendle and R. Schoen, Manifolds with 1/4-pinched curvature are space forms. J. Amer. Math. Soc. 22 (2009), 287-307.

[6] S. Brendle and R. Schoen, Classification of manifolds with weakly 1/4-pinched curvatures. Acta Math. 200 (2008), 1-13. MR.2386107

[7] B. Chow and D. Knopf, The Ricci flow: An introduction. Mathematical Surveys and Monographs, vol. 110, Amer. Math. Soc., Providence, RI, 2004. MR2061425(2005e:53101)

[8] B. Chow, P. Lu, and L. Ni, Hamilton's Ricci Flow. Graduate Studies in Mathematics, vol. 77, Amer. Math. Soc., Providence, RI, 2006; Science Press, New York, 2006. MR 2274812 (2008a:53068)

[9] O. Durumeric, A generalization of Berger's theorem on almost 1/4-pinched manifolds. II. J. Differential Geom. 26 (1987), no. 1, 101-139. MR892033(88m:53075)

[10] R. Hamilton, Three-manifolds with positive Ricci curvature. J. Diff. Geom. 17 (1982), 255306. MR664497 (84a:53050)

[11] R. Hamilton, Four-manifolds with positive curvature operator. J. Diff. Geom. 24 (1986), no. 2, 153-179. MR862046 (87m:53055)

[12] R. Hamilton, The Formation of Singularities in the Ricci Flow. Surveys in Differential Geometry, Vol. 2 (Cambridge, MA, 1993), Internat. Press, Cambridge, MA, 1995, 7-136. MR1375255(97e:53075) 
[13] P. Petersen, Riemannian Geometry, second edition, Springer-Verlag, New York, 2006. MR2243772 (2007a:53001)

[14] P. Petersen and F. Wilhelm, An exotic sphere with positive sectional curvature, arXiv:math.DG/0805.0812

[15] X. Rong, On the fundamental groups of manifolds of positive sectional curvature. Ann. of Math. (2) 143 (1996), no. 2, 397-411. MR1381991(97a:53067)

Department of Mathematics, University of California, 520 Portola Plaza, Los AnGeles, California 90095

E-mail address: petersen@math.ucla.edu

$U R L:$ http://www.math.ucla.edu/ ${ }^{\sim}$ petersen

Department of Mathematics, University of California, 520 Portola Plaza, Los AnGeles, California 90095

E-mail address: tao@math.ucla.edu

$U R L:$ http: //www. math.ucla.edu/ ${ }^{\sim}$ tao 